\title{
Correction to: A Novel Radio-Frequency Inductively Coupled Plasma Torch for Material Processing
}

\author{
Patrick Mirek $^{1} \cdot$ Sina Alavi ${ }^{1}$. Javad Mostaghimi ${ }^{1}$ \\ Published online: 4 October 2021 \\ (c) Springer Science+Business Media, LLC, part of Springer Nature 2021
}

\section{Correction to: Plasma Chemistry and Plasma Processing https://doi.org/10.1007/s11090-021-10200-8}

The original version of this article unfortunately contained a mistake. The corrected details are given below

a) The nomenclature given below was missed in the original paper.

\begin{tabular}{|c|c|c|c|}
\hline \multicolumn{4}{|c|}{ Nomenclature } \\
\hline $\operatorname{Re}$ & Reynolds nsumber & A & Vector potential (T-m) \\
\hline$\rho$ & Density $\left(\mathrm{kg} / \mathrm{m}^{3}\right)$ & $\mathrm{A}_{\theta}$ & Tangential component of vector potential (T-m) \\
\hline$v_{x}$ & Axial component of velocity $(\mathrm{m} / \mathrm{s})$ & $i$ & Imaginary unit number \\
\hline$v_{r}$ & Radial component of velocity (m/s) & $\mu_{0}$ & Vacuum magnetic permeability: $4 \pi \times 10^{-7}(\mathrm{H} / \mathrm{m})$ \\
\hline$v_{\theta}$ & Tangential component of velocity $(\mathrm{m} / \mathrm{s})$ & $\sigma$ & Electrical conductivity $(\mathrm{S} / \mathrm{m})$ \\
\hline$\mu$ & Dynamic viscosity (Pa-s) & $\omega$ & Angular frequency (rad/s) \\
\hline$F_{L, r}$ & Radial Component of Lorentz force $\left(\mathrm{N} / \mathrm{m}^{3}\right)$ & $\mathrm{J}_{\text {coil }}$ & Current density inside load coil $\left(\mathrm{A} / \mathrm{m}^{2}\right)$ \\
\hline$F_{p, x}$ & $\begin{array}{l}\text { Axial component of momentum source } \\
\text { term for particles }\left(\mathrm{N} / \mathrm{m}^{3}\right)\end{array}$ & B & Magnetic flux density $(\mathrm{T})$ \\
\hline$h$ & Enthalpy $(\mathrm{J} / \mathrm{kg})$ & $\mathrm{B}_{\mathrm{x}}$ & Axial component of magnetic flux density $(\mathrm{T})$ \\
\hline$k$ & Thermal conductivity (W/m-K) & $\mathrm{B}_{\mathrm{r}}$ & Radial component of magnetic flux density $(\mathrm{T})$ \\
\hline$c_{p}$ & Specific heat capacity $(\mathrm{J} / \mathrm{kg}-\mathrm{K})$ & $\mathbf{E}$ & Electric field intensity $(\mathrm{V} / \mathrm{m})$ \\
\hline$Q_{J H}$ & Joule heating source term $\left(\mathrm{W} / \mathrm{m}^{3}\right)$ & $\mathrm{E}_{\theta}$ & $\begin{array}{l}\text { Tangential component of electric field intensity } \\
(\mathrm{V} / \mathrm{m})\end{array}$ \\
\hline$\underline{Q_{R}}$ & Radiation sink term $\left(\mathrm{W} / \mathrm{m}^{3}\right)$ & $\mathrm{T}$ & Gas temperature $(\mathrm{K})$ \\
\hline
\end{tabular}

The original article can be found online at https://doi.org/10.1007/s11090-021-10200-8.

Javad Mostaghimi

mostag@mie.utoronto.ca

1 Centre for Advanced Coating Technologies, Department of Mechanical and Industrial Engineering

Faculty of Applied Science and Engineering, University of Toronto, 5 King's College Road,

Toronto, ON M5S 3G8, Canada 
Nomenclature

$Q_{p} \quad$ Energy source term for particles $\left(\mathrm{W} / \mathrm{m}^{3}\right) \quad \mathrm{P} \quad$ Gas pressure $(\mathrm{kPa})$

$T_{w}$ Torch wall temperature $(\mathrm{K})$

$k_{w}$ Torch wall thermal conductivity (W/m-k)

b) In Table 1: Geometric Parameter " $\mathrm{D}=1.5$ *" should be " $\mathrm{d}=1.5$ *" in order to match the case used in the text. The correct Table 1 is given below.

c) Page 8: Under heading "Conical Torch Design and Optimization Section", 3 rd line in the $3^{\text {rd }}$ paragraph: should be changed from "Inherent to the plasma as the Joule heating ..." to "Inherent to the plasma are the Joule heating ...".

Table 1 Geometric parameters for the model

\begin{tabular}{llll}
\hline Geometric Parameter & Value & Geometric Parameter & Value \\
\hline $\mathrm{L}_{1}(\mathrm{~mm})$ & 28.8 & $\beta$ (swirl velocity angle, $\left.{ }^{\circ}\right)$ & $22.5^{*}$ \\
$\mathrm{~L}_{2}(\mathrm{~mm})$ & 8 & $\mathrm{R}_{1}(\mathrm{~mm})$ & 28.8 \\
$\mathrm{~L}_{3}(\mathrm{~mm})$ & 100 & $\mathrm{R}_{2}(\mathrm{~mm})$ & 1.9 \\
$\mathrm{~L}_{4}(\mathrm{~mm})$ & 10 & $\mathrm{R}_{\mathrm{o}}(\mathrm{mm})$ & 20 \\
$\mathrm{~L}_{\mathrm{i}}(\mathrm{mm})$ & 31 & $\mathrm{R}_{\mathrm{c}}(\mathrm{mm})$ & 26.5 \\
$\mathrm{~L}_{\mathrm{ic}}(\mathrm{mm})$ & 14.5 & $\mathrm{D}_{\mathrm{c}}(\mathrm{mm})$ & 7 \\
$\alpha\left(^{\circ}\right)$ & $10^{*}$ & $\mathrm{~d}(\mathrm{~mm})$ & $1.5^{*}$ \\
$\mathrm{R}_{\mathrm{i}}(\mathrm{mm})$ & 13.8 & & \\
\hline
\end{tabular}

*Optimized value based on "Conical Torch Design and Optimization" section

Publisher's Note Springer Nature remains neutral with regard to jurisdictional claims in published maps and institutional affiliations. 